\title{
TERPENOIDS FROM LEAVES OF CALOCEDRUS FORMOSANA
}

\author{
Jim-Min Fang, Kuo-Chio Hsu and Yu-Shia Cheng* \\ Department of Chemistry, National Tawwan University, Täıeı, 10764, Taiwan, Republic of China
}

(Recelved in revised form 21 September 1988)

Key Word Index - Calocedrus formosana, Cupressaceae, leaves, labdane-type diterpenes, geranyl coumarate, epitorusolyl coumarate, $\gamma$-hydroxybutenolide diterpene

Abstract-Besides six labdane-type diterpenes, three new compounds of geranyl trans-coumarate, epitorusolyl ciscoumarate and diterpenold $\gamma$-hydroxybutenolide, were solated from leaves of Calocedrus formosana.

\section{INTRODUCTION}

The constituents of the heartwood of Calocedrus formosana, a member of Cupressaceae indigenous to Taiwan [1], have been extensively investigated in this laboratory [2-5]. In addition to a large quantity of terpenoid acids, such as shonanic, thujic and chaminic acids, the wood also contains tropolones, monoterpenes, naphthalenetype sesquiterpenes, diterpenord phenols, and lignans of hinokinin, hibalactone, and calocedrin We herein report the terpenoidal components isolated from leaves of this economically important tree

\section{RESULTS AND DISCUSSION}

By means of repetitıve chromatography and crystallızatıon, one monoterpenold derivative and eight diterpenes (Table 1) were isolated from $C$ formosana. Six known diterpenes were identıfied as phytol (2) $[6,7]$, transcommunic acid (3) $[8,9]$, 1socupressic acid $(5)[10]$, the acetate of isocupressic acid (6) [11], agatholal (7) [12,13], and pinosolide acid (8) [14-16] from their physical and spectroscopic properties (mp, $[\alpha]$, IR, MS, ${ }^{1} \mathrm{H}$ and ${ }^{13} \mathrm{C}$ NMR) Their pertinent ${ }^{13} \mathrm{CNMR}$ data are listed in Table 2 . The novel monoterpenord derivative $\left(\mathbf{M}^{+}\right.$at $m / z$ 300 ) was assigned as geranyl trans-coumarate (1) The IR absorptions at 1678 and $1629 \mathrm{~cm}^{-1}$, the UV absorption at $308 \mathrm{~nm}$, as well as the base peak at $m / z 147$, revealed that 1 contained a $p$-hydroxycinnamoyl moiety The trans-configuration was inferred from a large coupling constant of $160 \mathrm{~Hz}$ between two vinyl protons [17] The geranyl portion was determıned by comparıng the ${ }^{1} \mathbf{H}$ and ${ }^{13} \mathrm{C}$ NMR spectra with those of geraniol and geranyl acetate [18] The chemical shifts of C-4 $(\delta 39.45)$ and $C-10$ $(\delta 1648)$ for structure 1 are close to those of geranyl acetate $(\delta 3965$ and 1605$)$, but different from corresponding signals of neryl acetate ( $\delta 3134$ and 23.56) Saponification of compound 1 afforded geraniol and trans-coumaric acid, thus confirming the structural assignment of 1 .

Compound $4\left(\mathrm{M}^{+}\right.$at $\left.m / z 452\right)$ contained a coumaroyl molety as inferred from $\lambda_{\max }$ at $308 \mathrm{~nm}$ and the base peak at $m / z 147$ However, the double bond was in the cis configuration as two vinyl protons had a relatively small coupling constant of $12.6 \mathrm{~Hz}$, compared with $160 \mathrm{~Hz}$ in compound 1 [17]. This assignment was supported by saponification of $\mathbf{4}$ to give $c l s$-coumaric acid as a predominant product The other saponification product was found to be 13-epitorusolol (19-hydroxy-13-epimanool), mp $108-100^{\circ},[x]_{\mathrm{D}}^{25}+422^{\circ}$ (lit. $[12,19,20] 111-112^{\circ}$, $\left.[\alpha]_{\mathrm{D}}^{25}+423^{\circ}\right)$. The methylene protons at $\mathrm{C}-19$ ( $\delta 3.42$ and 365 , AB quartet) in 13-epitorusolol shifted to lower fields ( $\delta 388$ and 415 ) in ester 4 Thus, the ester linkage in 4 must be formed by the 19-hydroxyl group and ciscoumaric acid

The new diterpenoid $\gamma$-hydroxybutenolide 9, mp $175-176^{\circ}$, was determined as 16 -hydroxy-labda- $8(17), 13-$ diene-15,19-dioic acid butenolide from its spectroscopic properties Compound 9 actually comprised two C-16 epımers $\left(\begin{array}{ll}11 & 14\end{array}\right)$ as shown by two $\mathrm{H}-16$ resonances in the ${ }^{1} \mathrm{H}$ NMR spectrum at $\delta 594(\mathrm{br} \mathrm{s})$ and $597(\mathrm{br} s)$ as well as by two C-16 resonances at $\delta 98.8(d)$ and $99.3(d)$ The appropriate IR absorptions for carboxylic acid and conjugated $\gamma$-lactone appeared at 3000-2500, 1685 and $1728 \mathrm{~cm}^{-1}$ On treatment with a small amount of sodium methoxıde in $\mathrm{CD}_{3} \mathrm{OD}$, compound 9 underwent a ringopening reaction to exhibit a red shift of UV absorptions

Table $1 R_{f}$ values and percentage contents of terpenoids 1-9 obtained from Calocedrus tormosana

\begin{tabular}{llr}
\hline Compound & $R_{f}^{*}$ & Content $(\%)$ \\
\hline $\mathbf{1}$ & 054 & 117 \\
$\mathbf{2}$ & 069 & 083 \\
$\mathbf{3}$ & 047 & 2352 \\
$\mathbf{4}$ & 037 & 333 \\
$\mathbf{5}$ & 047 & 039 \\
$\mathbf{7}$ & 049 & 194 \\
$\mathbf{8}$ & 043 & 149 \\
$\mathbf{9}$ & 034 & 1254 \\
\hline
\end{tabular}

* The samples were run on a silica gel plate developed with ethyl acetatehexane ( 37$)$ 
<smiles>CC(C)=CCCC(C)=CCOC(=O)C=Cc1ccc(O)cc1</smiles>

1<smiles>C=C/C(C)=C/C[C@H]1C(=C)CC[C@H]2[C@@H](C(=O)O)CCC[C@]12C</smiles>

3<smiles>[R2]CC=C(C)CC[C@H]1C(=C)CC[C@H]2[C@@]([Y])(C([Y])=O)CCC[C@]12C</smiles>

$5 \quad \mathbf{R}=\mathrm{H}, \quad \mathrm{Y}=\mathrm{OH}$

$6 \mathrm{R}=\mathrm{Ac}, \mathrm{Y}=\mathrm{OH}$

$7 \mathrm{R}=\mathrm{Y}=\mathrm{H}$<smiles></smiles>

9<smiles>CC(C)CCCCCCC[C@H](C)CCCC(C)C</smiles>

2<smiles>C=C[C@H](O)CC[C@H]1C(=C)CC[C@H]2[C@@H](CC(=O)/C=C\c3ccc(O)cc3)CCC[C@]12C</smiles>

$\mathrm{HO}_{2} \mathrm{C}$

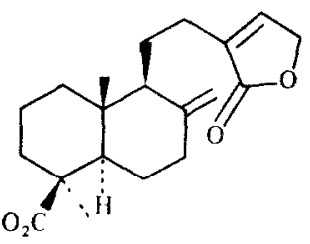

8 and to give a new signal in the ${ }^{1}$ H NMR spectrum at $\delta 854$ attributable to the resonance of an aldehyde proton [21]

In summary, three novel terpenoids 1,4 and 9 were isolated from $C$ formosana The major diterpenoidal components in leaves of this plant are trans-communic acid (3) and pinusolide acid (8)

\section{EXPERIMENTAL}

Plant material Calocedrus formosana (Florin) Florin was collected in the Tauchung county Leaves $(134 \mathrm{~kg})$ were arr-dried and exhaustively extracted with $\mathrm{Me}_{2} \mathrm{CO}$ After removal of solvent, the residue was extracted several times with hexane, followed by extraction with EtOAc The extractive of EtOAc was concd to give $188 \mathrm{~g}$ of dark brown viscous oil The oily sample was absorbed on $48 \mathrm{~g}$ of silica gel and subjected to $\mathrm{CC}$ (packed with $250 \mathrm{~g}$ of silica gel) with elution using a hexane-EtOAc gradient. Isolated compounds were further purified by crystallization or by HPLC on a Waters $\mu$-Porasil column
Geranyl trans-p-hydroxycunnamate (1) An only sample, $\mathrm{UV} \hat{i}_{\max }^{\mathrm{CHCl}} \mathrm{nm}(\varepsilon) 238(11750), 308(30200)$ IR $v_{\max }^{\text {neat }} \mathrm{cm}^{-1} 3347$. $1678,1629,1604,1585,1512,982,832$ MS $m / z$ (rel int.) 300 $[\mathrm{M}]^{+}(6), 231(10), 164(90), 147(100), 136(85), 121(80), 93(75), 92$ $(60), 80(80), 69(40), 41(50){ }^{1} \mathrm{H}$ NMR $\left(\mathrm{CDCl}_{3}, 300 \mathrm{MHz}\right) \delta 158$ (3H, s, Me-9), 166 (3H, s, Me-8), 171 (3H, s, Me-10), 202-211 $(4 \mathrm{H}, m), 472(2 \mathrm{H}, d . J=72 \mathrm{~Hz}), 507(1 \mathrm{H}, b r \mathrm{~s}, \mathrm{H}-6), 540(1 \mathrm{H}, t . J$ $=72 \mathrm{~Hz})_{,} 628\left(1 \mathrm{H}, d_{r} J=160 \mathrm{~Hz}\right) .685\left(2 \mathrm{H}, d_{r} J=86 \mathrm{~Hz}\right), 735$ $(2 \mathrm{H}, d, J=86 \mathrm{~Hz}), 762(1 \mathrm{H}, d, J=160 \mathrm{~Hz}){ }^{13} \mathrm{C} \mathrm{NMR}\left(\mathrm{CDCl}_{3}\right.$, $75 \mathrm{MHz})$ o $165(q, \mathrm{C}-10), 176(q, \mathrm{C}-8), 256(q, \mathrm{C}-9), 262(t, \mathrm{C}-5)$ $395(t, \mathrm{C}-4), 616(t, \mathrm{C}-1), 1150(d, \mathrm{C}-3), 1159\left(d, \mathrm{C}-6^{\prime}, \mathrm{C}-8^{\prime}\right), 1181$ $(d, C-6), 3237(d, C-2), 1267\left(s, C-4^{\prime}\right), 1300\left(d, C-5^{\prime}, C-9^{\prime}\right), 1318(s$. $C-7), 1425(s, C-3), 1450\left(d, C-2^{\prime}\right), 1583\left(s, C-7^{\prime}\right), 1682\left(s, C-1^{\prime}\right)$ Treatment of compound $\mathbf{l}$ with $\mathrm{KOH}$ by a similar procedure for saponification of 4 gave products of geraniol and trans-phydroxycinnamic acid

13S-Labda-8(17),14-diene-13-(c1s-p-hydroxycinnamoxy)-19-ol(4) An orly sample, $[\alpha]_{\mathrm{D}}^{25}+78\left(\mathrm{CHCl}_{3}, c 10\right) \mathrm{UV}_{\hat{\lambda}_{\max }}^{\mathrm{CHCl}_{3}} \mathrm{~nm}(\varepsilon)$ $240(10000), 308(13490)$ IR $v_{\max }^{\text {neat }} \mathrm{cm}^{-1} 3391,1709.1601,990$, $907 \mathrm{MS} m / z$ (rel int ) $452[\mathrm{M}]^{+}$(1) $434(5), 288(14), 270(25), 257$ 
Table $2{ }^{13} \mathrm{C}$ NMR spectral data of compounds $3-9\left(\mathrm{CDCl}_{3}, 75 \mathrm{MHz}, \delta\right)$

\begin{tabular}{rrrrrrrr}
\hline $\mathbf{C}$ & \multicolumn{1}{c}{$\mathbf{3}$} & $\mathbf{4}$ & $\mathbf{5}$ & $\mathbf{6}$ & \multicolumn{1}{c}{$\mathbf{7}$} & $\mathbf{8}$ & \multicolumn{1}{c}{$\mathbf{9}$} \\
\hline 1 & 392 & 394 & 391 & 389 & 382 & 391 & 392 \\
2 & 198 & 178 & 209 & 197 & 190 & 198 & 210 \\
3 & 384 & 340 & 378 & 377 & 341 & 386 & 386 \\
4 & 441 & 442 & 441 & 440 & 484 & 442 & 445 \\
5 & 563 & 560 & 562 & 562 & 557 & 562 & 56.6 \\
6 & 258 & 244 & 260 & 259 & 238 & 259 & 26.7 \\
7 & 377 & 360 & 383 & 382 & 34.1 & 378 & 378 \\
8 & 1478 & 1471 & 1478 & 1476 & 1470 & 1473 & 1472 \\
9 & 562 & 560 & 554 & 552 & 545 & 556 & 563 \\
10 & 403 & 394 & 403 & 402 & 397 & 504 & 411 \\
11 & 233 & 205 & 227 & 216 & 217 & 218 & 211 \\
12 & 1415 & 387 & 386 & 385 & 381 & 289 & 268 \\
13 & 1334 & 746 & 1402 & 1426 & 1393 & 1439 & 170.2 \\
14 & 1338 & 1436 & 1228 & 1179 & 1232 & 134.8 & 1171 \\
15 & 1099 & 1156 & 591 & 613 & 588 & 701 & 1702 \\
16 & 118 & 272 & 163 & 163 & 161 & 1744 & $993^{*}$ \\
17 & 1076 & 1133 & 1064 & 1063 & 1070 & 1068 & 1068 \\
18 & 290 & 232 & 289 & 288 & 241 & 289 & 289 \\
19 & 1837 & 667 & 1833 & 1839 & 2056 & 1838 & 1831 \\
20 & 128 & 177 & 127 & 126 & 133 & 127 & 127 \\
\hline
\end{tabular}

* Signal for the major isomer The corresponding sıgnal of the minor isomer appeared at $\delta 988$

(17), $189(44), 164(6), 147(100), 121(7){ }^{1} \mathrm{H}$ NMR (CDCl $), 8074$ $(3 \mathrm{H}, s, \mathrm{Me}-10), 087(3 \mathrm{H}, s, \mathrm{Me}-4), 1.08(3 \mathrm{H}, s, \mathrm{Me}-13), 3.88(1 \mathrm{H}, d$, $J=110 \mathrm{~Hz}), 415(1 \mathrm{H}, d, J=110 \mathrm{~Hz}), 493(1 \mathrm{H}, b r s, \mathrm{H}-17), 4.98$ $(1 \mathrm{H}$, br s, H-17), $501(1 \mathrm{H}, b r d, J=110 \mathrm{~Hz}), 524(1 \mathrm{H}, b r d, J$ $=176 \mathrm{~Hz}), 574(1 \mathrm{H}, d, J=126 \mathrm{~Hz}), 629(1 \mathrm{H}, d d, J=176$, $110 \mathrm{~Hz}), 675(2 \mathrm{H}, d, J=80 \mathrm{~Hz}), 677(1 \mathrm{H}, d, J=126 \mathrm{~Hz}), 7.52$ $(2 \mathrm{H}, d, J=80 \mathrm{~Hz})$ Compound $4(52 \mathrm{mg})$ was treated with $\mathrm{KOH}$ ( $1 \mathrm{~N}$ in $4 \mathrm{ml}$ of $95 \%$ EtOH) After reflux for $5 \mathrm{hr}$, the mixture was diluted with $\mathrm{H}_{2} \mathrm{O}$ and extracted with $\mathrm{Et}_{2} \mathrm{O}$ The ethereal phase was concentrated to afford $34 \mathrm{mg}$ of colourless solids 1dentical to 13-epitorulosol The aq phase was acidified with $\mathrm{HCl}$ and extracted with $\mathrm{Et}_{2} \mathrm{O}$ to give $18 \mathrm{mg}$ of $p$-hydroxycinnamic acid as a mixture of $c t s$ - and trans-1somers in a ratio of 31

16-Hydroxy-labda-8(17),13-diene-15,19-diolc actd butenolde (9) UV $\lambda_{\max }^{\mathrm{EtOH}} \mathrm{nm}(\varepsilon) 208$ (2372); in the presence of $\mathrm{KOH}, 204$ (5472), 228 (3257), 254 (16i2) IR $v_{\max }^{\mathbf{K B r}} \mathrm{cm}^{-1} 3373,3000-2500$, $1728,1685,1207,1139,890 \mathrm{MS} m / z$ (rel int ) $348[\mathrm{M}]^{+}(6), 330$ (22), 284 (10), 235 (28), 189 (38), 167 (46), 121 (100), 105 (18), 81

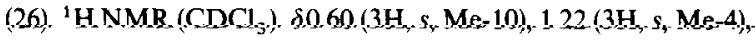
$448(1 \mathrm{H}, b r s), 488(1 \mathrm{H}, b r s), 5.83(1 \mathrm{H}, b r s), 594 / 597$ (br s, OCHO) ${ }^{13} \mathrm{C}$ NMR (acetone- $d_{6}$ ) $\delta 131,20.7,217,270,27.3$, $290,38,9.39 .3,398,4,1,4,44,5,56,4,56,5,996,100,0,106,8,117,2$, $1488,1713,1788$.

Acknowledgements - We are grateful to the National Science Councl (ROC) for financial support, and to Ms Chia-Yu Huang for technical assistance

\section{REFERENCES}

1. Flora of Taiwan (1975) Vol 1, p 538, Epoch, Tawan

2 Cheng, Y S. and Lin, K C. (1970) Chemistry (Chinese) 28.
3 Cheng, Y S and Lin, K C (1971) Chemustry (Chunese) 94.

4. Fang, J. M., Jan, S T and Cheng, Y S (1985) Phytochemistry 24, 1863

5 Fang, J M, Jan, S. T and Cheng, Y.S (1987) Phytochemstry 26, 853 .

6 Schmıd, M, Gerber, F and Hirth, G (1982) Helv Chım. Acta 65, 684

7 Burrell, J. W K, Garwood, R F, Jackman, L. M., Oskay, E. and Weedon, B C L (1966) J Chem Soc C, 2144.

8 Thomas, B R (1966) Acta Chem Scand. 20, 1074

9 Lee, G. H, Lin, C C., Cheng, Y. S and Pen, S. M (1987) Acta Cryst C43, 1382

10. Cambie, R C, Grigor, B A and Tiong, M I. (1981) Aust J. Chem 34, 1073

11 Caputo, R, Mangoni, L and Previtera, L (1974) Phytochemistry 13, 471

12. Rowe, J W and Shaffer, G. W (1965) Tetrahedron Letters 2633

13 Rowe, J W, Scroggins, J H (1964) J Ory Chem 29, 1554.

14. Baldugan. V A. Lusina, A. I, Kashatonnva, N. K.. and Pentegova, V A. (1970) Khim. Priod Soedin. 541, (1971) Chem Abstr 74, 84003s

1.5 Gough, L. L. and Mulls, L.S (1974), Phytoshemustry 13, 1612

16. Rojas, E T. and Rodriguez, H L (1978) Phytochemistry 17, 574

17 Shıbuya, T., Funamızu, M. and Kitahara, Y. (1978) Phytochemistry 17, 979

18. de Haan, J W. and van de Ven, L J M. (1971) Tetrahedron Letters 2703

19. Bruns, K. (1970) Tetrahedron Letters 3263

20. Manning, T D. R (1973) Aust. J Chem 26, 2735

21 Midland, S L., Wing, R. M and Sims, J. J (1983) J. Org Chem. 48, 1906 